\title{
Bedaquiline in the treatment of multidrug- and extensively drug- resistant tuberculosis
}

\author{
Alexander S. Pym ${ }^{1}$, Andreas H. Diacon², Shen-Jie Tang ${ }^{3}$, Francesca Conradie ${ }^{4}$, \\ Manfred Danilovits ${ }^{5}$, Charoen Chuchottaworn ${ }^{6}$, Irina Vasilyeva ${ }^{7}, K_{\text {Koen Andries }}{ }^{\prime}$, \\ Nyasha Bakare9, Tine De Marez ${ }^{9}$, Myriam Haxaire-Theeuwes ${ }^{8}$, Nacer Lounis ${ }^{8}$, \\ Paul Meyvisch ${ }^{8}$, Ben Van Baelen ${ }^{8}$, Rolf P.G. van Heeswijk ${ }^{8}$ and \\ Brian Dannemann ${ }^{9}$ on behalf of the TMC207-C209 Study Group ${ }^{10}$
}

\begin{abstract}
Affiliations: ${ }^{1}$ Medical Research Council and Kwazulu Research Institute for TB and HIV (K-RITH), Durban, South Africa. ${ }^{2}$ Division of Medical Physiology and Dept of Science and Technology/National Research Foundation Centre of Excellence for Biomedical Tuberculosis Research and Medical Research Council Centre for Tuberculosis Research, Faculty of Medicine and Health Sciences, University of Stellenbosch, Cape Town, South Africa. ${ }^{3}$ Tuberculosis Dept, Beijing Chest Hospital, Capital Medical University, Beijing Tuberculosis and Thoracic Tumour Research Institute, Beijing, China. ${ }^{4}$ Clinical HIV Research Unit, Dept of Medicine, Faculty of Health Sciences, University of the Witwatersrand, Johannesburg, South Africa. ${ }^{5}$ Tartu University Lung Hospital, Tartu, Estonia. ${ }^{6}$ Chest Disease Institute, Ministry of Public Health, Nonthaburi, Thailand. ${ }^{7}$ Central Tuberculosis Research Institute, Russian Academy of Medical Sciences, Moscow, Russia. ${ }^{8}$ Janssen Infectious Diseases BVBA, Beerse, Belgium. ${ }^{9}$ Janssen Research \& Development LLC, Titusville, NJ, USA. ${ }^{10}$ Members of the TMC207-C209 Study Group are listed in the Acknowledgements section.
\end{abstract}

Correspondence: Alexander S. Pym, Kwazulu Research Institute for TB and HIV (K-RITH), K-RITH Tower Building, Level 3, Nelson R. Mandela School of Medicine, 719 Umbilo Road, Durban 4001, South Africa.

E-mail: Alex.Pymak-rith.org

ABSTRACT Bedaquiline, a diarylquinoline, improved cure rates when added to a multidrug-resistant tuberculosis (MDR-TB) treatment regimen in a previous placebo-controlled, phase 2 trial (TMC207-C208; NCT00449644). The current phase 2, multicenter, open-label, single-arm trial (TMC207-C209; NCT00910871) reported here was conducted to confirm the safety and efficacy of bedaquiline.

Newly diagnosed or previously treated patients with MDR-TB (including pre-extensively drug-resistant (pre-XDR)-TB or extensively drug-resistant (XDR)-TB) received bedaquiline for 24 weeks with a background regimen of anti-TB drugs continued according to National TB Programme treatment guidelines. Patients were assessed during and up to 120 weeks after starting bedaquiline.

Of 233 enrolled patients, $63.5 \%$ had MDR-TB, 18.9\% had pre-XDR-TB and 16.3\% had XDR-TB, with $87.1 \%$ having taken second-line drugs prior to enrolment. 16 patients (6.9\%) died. 20 patients $(8.6 \%)$ discontinued before week 24, most commonly due to adverse events or MDR-TB-related events. Adverse events were generally those commonly associated with MDR-TB treatment. In the efficacy population $(\mathrm{n}=205)$, culture conversion (missing outcome classified as failure) was $72.2 \%$ at 120 weeks, and $73.1 \%$, $70.5 \%$ and $62.2 \%$ in MDR-TB, pre-XDR-TB and XDR-TB patients, respectively.

Addition of bedaquiline to a background regimen was well tolerated and led to good outcomes in this clinically relevant patient cohort with MDR-TB.

@ERSpublications

Bedaquiline safety data in a broad patient population treated for drug-resistant TB including XDR-TB (C209 study) http://ow.ly/TcZBh

For editorial comment see Eur Respir J 2016; 47: 394-402 [DOI: 10.1183/13993003.01891-2015].

This article has supplementary material available from erj.ersjournals.com

Received: May 082015 | Accepted after revision: Oct 022015 | First published online: Dec 032015

Copyright OERS 2016 


\section{Introduction}

Multidrug-resistant tuberculosis (MDR-TB), defined as disease caused by strains of Mycobacterium tuberculosis resistant to at least isoniazid and rifampicin, threatens to erode progress in the global control of TB [1]. Drug resistance may worsen further with the emergence of resistance to at least one of the injectable agents (amikacin, kanamycin, capreomycin) or to fluoroquinolones [2] (deemed pre-extensively drug-resistant (pre-XDR)-TB by some authors [3]) or both (extensively drug-resistant (XDR)-TB). An estimated 480000 cases of MDR-TB occurred in 2013, of which 9\% were XDR-TB [1]. It has been reported by others that $\sim 50 \%$ of patients with MDR-TB respond favourably to treatment $[1,4]$, acknowledging this proportion might be higher with improved case holding and improved surveillance in the countries included in pooled datasets. Response rates in patients with XDR isolates are very poor $[1$, 5-8]. Treatment outcomes in 1269 XDR-TB patients from 40 countries as reported by the World Health Organization (WHO) were poor, with 35\% mortality, $10 \%$ failures, $33 \%$ lost to follow-up and only $22 \%$ completing treatment successfully [1]. New drugs are urgently required to improve current regimens [1].

Bedaquiline, a diarylquinoline compound with a novel mechanism of anti-TB action [9], has been granted accelerated or conditional approval in the USA (2012) and Europe (2014) for use in MDR-TB, with interim guidance for its use provided by the WHO [10]. In a randomised, placebo-controlled trial (TMC207-C208), 24 weeks of bedaquiline treatment significantly increased the culture conversion rate in patients with MDR-TB [11]. The overall incidence of adverse events (AEs) was similar, but a greater number of deaths was seen in the bedaquiline group than in the placebo group (10 (12.7\%) versus 2 (2.5\%), respectively) [11]. Based on analyses of interim results of stage 2 of C208, bedaquiline was made available for compassionate use in 2011 prior to its regulatory approval in now 40 countries. Several groups have recently reported their experience from the compassionate-use programmes, which have provided access to $>700$ patients worldwide [12-15]. In addition, a collaboration with the US Agency for International Development is making bedaquiline available free of charge to $>100$ global-fund-eligible countries over 4 years.

The objective of this study (TMC207-C209) was to evaluate the safety and tolerability as well as efficacy of bedaquiline in combination with a background regimen (BR) for the treatment of a broader cohort of patients with drug-resistant-TB, including pre-XDR and XDR-TB. Here, we report the final safety and efficacy data of this phase 2, open-label trial and discuss this in relation to other bedaquiline studies for MDR-TB [11, 16-17].

\section{Methods}

\section{Participants}

Participants were recruited at 31 sites in 11 countries in Asia (China, South Korea, Philippines, Thailand) and Eastern Europe (Estonia, Latvia, Russia, Turkey, Ukraine), as well as in Peru and South Africa.

We recruited patients aged $\geqslant 18$ years with pulmonary MDR-TB, who had sputum smear or culture positive for M. tuberculosis, and had confirmed resistance to isoniazid and rifampicin by culture-based drug susceptibility testing (DST) or by a rapid nucleic acid screening test in the preceding 6 months. Patients with XDR-TB were eligible if their isolates were likely to be susceptible to three or more anti-TB drugs in their BR [18]. HIV-infected patients with $\mathrm{CD}^{+}$cell count $<250$ cells $\cdot \mathrm{mm}^{-3}$ or with AIDS-defining illnesses other than TB were excluded. HIV patients on antiretroviral therapy (ART) were eligible if they met specific criteria, but none were enrolled. Exclusion criteria included complicated or severe extrapulmonary manifestations of TB; expected surgery for TB within the next 6 months; significant cardiac arrhythmia requiring medication; Fridericia-corrected QT (QTcF) interval $>450 \mathrm{~ms}$; history or risk factors for Torsades de Pointes; concomitant medications that prolong the QT/QTc interval, including moxifloxacin and gatifloxacin; drug hypersensitivity; alcohol or drug abuse; pregnancy or breastfeeding; and prior receipt of bedaquiline.

\section{Study design}

This was a phase 2, multicenter, open-label, single-arm trial (www.clinicaltrials.gov: NCT00910871) (online supplementary figure S1). Participants received $400 \mathrm{mg}$ bedaquiline once daily for 2 weeks then

Support statement: This study was supported by Janssen Pharmaceuticals. The study sponsor was involved in the design and conduct of the trial, and in the collection and analysis of the data. The corresponding author had full access to the clinical trial report and final responsibility to submit the manuscript for publication. Funding information for this article has been deposited with FundRef.

Clinical trial: This study is registered at www.clinicaltrials.gov with identifier number NCT00910871.

Conflict of interest: Disclosures can be found alongside the online version of this article at erj.ersjournals.com 
$200 \mathrm{mg} 3$ times a week for a further 22 weeks in combination with a BR of drugs chosen by the investigator in accordance with National TB Programme (NTP) guidelines. Bedaquiline tablets were taken after breakfast with water. After bedaquiline treatment, participants were followed for a further 96 weeks during which BR was completed (online supplementary figure S1). Intake of bedaquiline and BR was supervised using directly observed treatment according to NTP guidelines. Patients who prematurely discontinued were considered treatment failures, but were followed for collection of survival data (unless consent was withdrawn) until the end of the trial.

\section{Study assessments}

After screening, clinic visits were scheduled at baseline, after 1 day (start of bedaquiline treatment), and at $2,4,8,12,16,20$ and 24 weeks. All patients were then followed for 96 weeks with clinic visits every 12 weeks.

At each visit, patients had a physical examination, assessment for AEs, vital signs and an ECG. HIV-positive patients had $\mathrm{CD}^{+}{ }^{+}$cell and viral load monitoring. Determination of laboratory parameters was performed at screening, baseline, and after 4, 12 and 24 weeks, and all subsequent visits. The Division of Microbiology and Infectious Diseases adult toxicity tables were used for grading the severity of AEs [19]. Specific AEs of interest (based on nonclinical and clinical data for bedaquiline) were identified by probing the database with Standardised MedDRA Queries [20] for acute pancreatitis, rhabdomyolysis/ myopathy, severe cutaneous AEs and Torsades de Pointes/QT prolongation, and selected sub-Standardised MedDRA Queries for drug-related hepatic disorders (see footnote to table 4).

Treatment efficacy was assessed using triplicate spot sputum samples at every visit (except day of first dose) by liquid culture using mycobacteria growth indicator tubes (MGIT; Becton Dickinson, Sparks, MD, USA).

DST (carried out at the Institute of Tropical Medicine, Antwerp, Belgium), for bedaquiline and other anti-TB drugs, was performed at baseline and after 24 weeks of bedaquiline treatment, and in patients reverting to culture-positive. The minimal inhibitory concentration (MIC) of bedaquiline determined on 7H11 agar was defined as the lowest concentration $\left(\mu \mathrm{g} \cdot \mathrm{mL}^{-1}\right)$ that prevented growth of $M$. tuberculosis by $99 \%$.

\section{Study end-points and statistical methods}

A sample size of $\sim 225$ patients was considered sufficient to allow for meaningful conclusions on the safety of bedaquiline because any $\mathrm{AE}$ expected to occur in a single patient with a probability of $1 \%$ would be observed with a $90 \%$ probability.

Safety analyses were conducted on the intent-to-treat (ITT) population, which included all randomised patients who had at least one intake of bedaquiline. The efficacy analyses were performed on the modified ITT (mITT) population that excluded patients with drug-susceptible TB or patients with negative cultures at screening and/or baseline.

The primary efficacy end-point was the time to confirmed sputum culture conversion (two consecutive visits with negative MGIT cultures from spot sputa collected at least 25 days apart and not followed by a confirmed positive culture) during 24 weeks of bedaquiline treatment. Patients with no culture conversion, who died or who discontinued before the considered time point were classified as nonresponders (missing=failure), irrespective of their culture status at time of dropout or death and the time to culture conversion was censored at their last assessment. Cure rates were also assessed on the basis of the WHO outcomes for MDR-TB [21] with the modification that definition of completion of treatment and cure were determined based on completing the study rather than completing treatment.

\section{Ethics}

Local ethics committees approved the protocol at all study sites. The study was conducted in accordance with the principles of the Declaration of Helsinki and Good Clinical Practice, and all patients (or their representatives) provided written informed consent.

\section{Results}

Study population

Of 294 patients screened between August 20, 2009 and September 27, 2010, 233 were enrolled and included in the ITT (safety) population (online supplementary figure S2). Of these, 179 patients $(76.8 \%)$ completed the study and 54 patients (23.2\%) discontinued, with 17 (7.3\%) patients discontinuing due to AEs including MDR-TB-related events. 32 of the 179 (17.9\%) ITT patients who completed the trial were still receiving treatment with BR at study end. The mITT (efficacy) population, which excluded three patients with drug-susceptible TB and 25 with negative cultures at screening and/or baseline, comprised 205 patients. The 
characteristics of the safety and efficacy populations were similar (table 1). Regarding the safety population ( $\mathrm{n}=233), 39.9 \%$ (93/233) had MDR-TB (excluding pre-XDR-TB and XDR-TB), 23.6\% (55/233) had resistance to isoniazid and rifampicin MDR-TB, but pre-XDR or XDR status was not determined, $18.9 \%$ (44/233) had pre-XDR-TB and 16.3\% (38/233) had XDR-TB. Most patients were HIV-negative (96.0\% of those tested), had cavitary disease (63.5\%) and had received second-line anti-TB drugs (87.1\%) for a median of 36 days (range 6-2647 days) prior to enrolment (table 1). Of the eight HIV-positive patients in the efficacy population, three were infected with MDR-TB, three with pre-XDR-TB and one with XDR-TB; one patient had MDR-TB for which the pre-XDR or XDR status was not determined. Table 2 shows the most common BR drugs for the efficacy population $(n=205)$. Regarding other BR drugs of note, clofazimine was received by 13 patients at baseline and 36 patients during the 120 -week study, and linezolid was received by 12 and 34 patients, respectively. No patients received delamanid during the trial.

\begin{tabular}{|c|c|c|}
\hline & $\begin{array}{l}\text { Safety (ITT) population } \\
\qquad N=233\end{array}$ & $\begin{array}{l}\text { Efficacy (mITT) population } \\
\qquad \mathrm{N}=205\end{array}$ \\
\hline Male & $150(64.4)$ & $132(64.4)$ \\
\hline Age years & $32(18-68)$ & $32(18-68)$ \\
\hline \multicolumn{3}{|l|}{ Race } \\
\hline American Indian" & $8(3.4)$ & $6(2.9)$ \\
\hline Asian & 89 (38.2) & $84(41.0)$ \\
\hline Black or African American & 75 (32.2) & $67(32.7)$ \\
\hline White & $61(26.2)$ & $48(23.4)$ \\
\hline Bodyweight kg & $57(30-113)$ & $57(30-113)$ \\
\hline Body mass index $\mathrm{kg} \cdot \mathrm{m}^{-2}$ & $19.9(13-37)$ & $19.8(13-37)$ \\
\hline \multicolumn{3}{|l|}{ Lung cavitations } \\
\hline None or $<2 \mathrm{~cm}$ & 85 (36.5) & $70(34.1)$ \\
\hline Cavitations $\geqslant 2 \mathrm{~cm}$ in one lung only & $120(51.5)$ & $108(52.7)$ \\
\hline Cavitations $\geqslant 2 \mathrm{~cm}$ in both lungs & $28(12.0)$ & $27(13.2)$ \\
\hline \multicolumn{3}{|l|}{ Extent of resistance of $M$. tuberculosis strain } \\
\hline Drug-susceptible TB & $3(1.3)$ & 0 \\
\hline MDR-TB & $148(63.5)$ & $124(60.5)$ \\
\hline Pre-XDR-TB & $44(18.9)$ & $44(21.5)$ \\
\hline Pre-XDR-TB fluoroquinolone resistant & $31(13.3)$ & $31(15.1)$ \\
\hline Pre-XDR-TB injectable resistant & $13(5.6)$ & $13(6.3)$ \\
\hline XDR-TB & 38 (16.3) & 37 (18.0) \\
\hline \multicolumn{3}{|l|}{ Previous TB medications } \\
\hline No previous anti-TB treatment & $12(5.2)$ & $12(5.9)$ \\
\hline Previous use of second-line TB drugs & $203(87.1)$ & $177(86.3)$ \\
\hline \multicolumn{3}{|l|}{ Previous treatment with } \\
\hline Aminoglycosides & $156(67.0)$ & $139(67.8)$ \\
\hline Fluoroquinolones & $193(82.8)$ & $167(81.5)$ \\
\hline Macrolides & $33(14.2)$ & $33(16.1)$ \\
\hline \multicolumn{3}{|l|}{ Pooled centres } \\
\hline Asia (other) & $33(14.2)$ & $31(15.1)$ \\
\hline China & $51(21.9)$ & $50(24.4)$ \\
\hline Eastern Europe & 52 (22.3) & $41(20.0)$ \\
\hline South Africa & 79 (33.9) & $70(34.1)$ \\
\hline South America & $18(7.7)$ & $13(6.3)$ \\
\hline HIV status & $\mathrm{N}=225$ & $N=198$ \\
\hline Positive & $9(4.0)$ & $8(4.0)$ \\
\hline
\end{tabular}

Data are presented as $\mathrm{n}(\%)$ or median (range). $\mathrm{n}$ : number of patients with result; $\mathrm{N}$ : number of patients with data; ITT: intent-to-treat; mITT: modified intent-to-treat; TB: tuberculosis; $M$. tuberculosis: Mycobacterium tuberculosis; MDR-TB: multidrug-resistant TB; pre-XDR-TB: pre-extensively drug-resistant TB (MDR-TB and resistant to either any second-line injectable drug lamikacin, kanamycin and capreomycin) or any fluoroquinolone); XDR-TB: extensively drug-resistant TB (MDR-TB and resistant to any second-line injectable drug (amikacin, kanamycin and capreomycin) and any fluoroquinolone). \#: race based on US Food and Drug Administration template (no patients from North America were randomised); ๆ: for 55 patients in the safety population (31 patients in the efficacy population), the extent of resistance of the M. tuberculosis strain could not be determined due to missing baseline central drug susceptibility test results (these patients were considered to have MDR-TB based on previous drug-susceptibility testing). 


\begin{tabular}{lcc}
$\begin{array}{l}\text { TABLE } 2 \text { Background regimen at baseline and during the } 120 \text { week study lefficacy (modified } \\
\text { intent-to-treat) population) }\end{array}$ & $\begin{array}{c}\text { During the } \mathbf{1 2 0} \text {-week study } \\
\mathbf{N}=\mathbf{2 0 5}\end{array}$ \\
$\begin{array}{l}\text { Most frequently used anti-TB drugs in the } \\
\text { background regimen }\end{array}$ & $\begin{array}{c}\text { Baseline } \\
\mathbf{N}=\mathbf{2 0 5}\end{array}$ & $185(90.2)$ \\
\hline $\begin{array}{l}\text { Fluoroquinolones } \\
\text { Ofloxacin }\end{array}$ & $180(87.8)$ & $102(49.8)$ \\
$\quad$ Levofloxacin & $101(49.3)$ & $79(38.5)$ \\
Ethionamide or protionamide & $66(32.2)$ & $175(85.4)$ \\
Pyrazinamide & $160(78)$ & $156(76.1)$ \\
Aminoglycosides & $152(74.1)$ & $154(75.1)$ \\
Terizidone or cycloserine & $152(74.1)$ & $130(63.4)$ \\
Pas-c & $113(55.1)$ & $123(60)$ \\
Ethambutol & $97(47.3)$ & $117(57.1)$
\end{tabular}

Data are presented as n (\%). TB: tuberculosis. " : 20/205 (including seven with an extensively drug-resistant isolatel patients did not receive a fluoroquinolone during the 120-week study; gatifloxacin was received by one patient at baseline and one patient during the 120-week study; moxifloxacin was received by one and 29 patients, respectively; ": excludes capreomycin.

\section{Adverse events}

The most common AEs (table 3) were similar to those frequently described in MDR-TB treatment cohorts $[22,23]$ and most were grade 1 or 2 [19]. Hyperuricaemia $(9.4 \%)$ and increased aspartate aminotransferase (AST) $(3.4 \%)$ were the most frequent grade $\geqslant 3$ AEs. The incidence of AEs was highest during the first 12 weeks of the trial. Serious AEs were reported in 47 (20.2\%) patients; of these, respiratory infections/ disorders were the most common (online supplementary table S1).

There were 16 deaths (6.9\% of 233 patients) (table 4), four of which occurred after withdrawal from the study. There was no pattern to the deaths (figure 1). Only one of the HIV co-infected patients in the trial died. This patient had an XDR strain isolated at baseline. Three deaths (from renal impairment and two from TB) occurred during bedaquiline treatment. Nine of 16 causes of deaths as reported by the investigator were due to TB or a TB-related illness and eight of these had not converted or reverted to culture-positive. A further four deaths were due to respiratory disorders. The 16 deaths were considered not related to bedaquiline (table 4).

AEs of prolongation of the QTcF interval were reported infrequently (table 3). The two patients with an increase in QTcF interval $>500 \mathrm{~ms}$ were both taking clofazimine and one had concurrent hypokalaemia. No episodes of clinically significant dysrthymia were reported. The mean maximum change in QTcF interval from baseline was $14.2 \mathrm{~ms}$. This fell to $<10 \mathrm{~ms}$ after bedaquiline was stopped (figure 2 ). In the patients taking clofazimine at week $24(\mathrm{n}=17)$, the mean maximum change in QTcF interval from baseline to week 24 was $31.9 \mathrm{~ms}$ compared with $12.3 \mathrm{~ms}$ for those not taking clofazimine $(\mathrm{n}=177)$.

Elevations in transaminases were reported. One patient taking multiple background drugs with potential for hepatotoxicity met the laboratory criteria for Hy's law at week 32 (treatment-emergent serum alanine aminotransferase (ALT) or AST $>3$ times upper limit of normal with concurrent serum total bilirubin $>2$ times upper limit of normal) [24] and recovered. Most of the grade $\geqslant 3$ increases ( $\geqslant 3$ times upper limit of normal) in treatment-emergent AST or ALT (table 3) had onset after 24 weeks of bedaquiline treatment. In a more detailed analysis over 120 weeks, hepatic-related AEs were identified in 42 patients (18.0\%), predominantly increases in AST/ALT (table 3), and considered at least possibly related to bedaquiline by the investigator in four patients $(1.7 \%)$. One of these four did not resolve, but the patient also had hepatitis C infection. Grade 3 or 4 hepatic-related AEs were identified in 15 (6.4\%) patients, but considered at least possibly related to bedaquiline in only one.

\section{Microbiological outcomes}

The time to culture conversion (missing=failure) is shown in figure 3. The median time based on 24-week data was 57 days. The sputum culture conversion rate was $79.5 \%$ (163/205 patients) at 24 weeks and $72.2 \%$ (148/205 patients) at 120 weeks (figure 4). Culture conversion at 24 weeks was durable and associated with a high likelihood of response at 120 weeks: $85.3 \%$ of 24 -week responders (139/163 patients) were still responders at 120 weeks. The majority of those classified as nonresponders at week 120 had discontinued after culture conversion (19/24), with five reverting to culture-positive after early conversion. Nine out of 42 nonresponders at 24 weeks responded by week 120 . 
TABLE 3 Incidence of treatment-emergent adverse events (AEs) and grade $\geqslant 3$ laboratory abnormalities (safety (intent-to-treat) population)

\begin{tabular}{|c|c|c|}
\hline AE category & $\begin{array}{c}24 \text { weeks of } \\
\text { bedaquiline treatment } \\
N=233\end{array}$ & $\begin{array}{c}\text { Overall } 120 \text { weeks } \\
\text { of treatment } \\
N=233\end{array}$ \\
\hline Any AE & 212 (91.0) & 219 (94.0) \\
\hline Any serious $\mathrm{AE}$ & $15(6.4)$ & $47(20.2)$ \\
\hline Any $A E$ related to $T B$ & $47(20.2)$ & 77 (33.0) \\
\hline Any grade $4 \mathrm{AE}$ & $5(2.1)$ & $24(10.3)$ \\
\hline Any $A E$ possibly related to bedaquiline & $77(33.0)$ & $77(33.0)$ \\
\hline Any serious $A E$ possibly related to bedaquiline & $1(0.4)$ & $1(0.4)$ \\
\hline Any $\mathrm{AE}$ leading to bedaquiline discontinuation & $6(2.6)^{\#}$ & $6(2.6)^{\#}$ \\
\hline Any $A E$ leading to background regimen discontinuation & $49(21.0)$ & 73 (31.3) \\
\hline \multicolumn{3}{|l|}{ AEs regardless of causality with $>10 \%$ incidence } \\
\hline Hyperuricaemia & $49(21.0)$ & $56(24.0)$ \\
\hline Nausea & $27(11.6)$ & $35(15.0)$ \\
\hline Arthralgia & $29(12.4)$ & $35(15.0)$ \\
\hline Headache & $21(9.0)$ & 31 (13.3) \\
\hline Diarrhoea & $18(7.7)$ & $27(11.6)$ \\
\hline Vomiting & $21(9.0)$ & $27(11.6)$ \\
\hline \multicolumn{3}{|l|}{ AEs of special interest ${ }^{\text {I }}$} \\
\hline Hepatic disorders & $27(11.6)$ & $42(18.0)$ \\
\hline Increased AST & $9(3.9)$ & $14(6.0)$ \\
\hline Increased ALT & $5(2.1)$ & $12(5.2)$ \\
\hline QT prolongation ${ }^{+}$ & $6(2.6)$ & $10(4.3)$ \\
\hline Severe cutaneous AEs & $8(3.4)$ & $9(3.9)^{\S}$ \\
\hline Acute pancreatitis $f$ & $3(1.3)$ & $7(3.0)$ \\
\hline $\begin{array}{l}\text { Laboratory abnormalities grade } \geqslant 3 \text { with }>3 \% \text { incidence } \\
\text { during } 120 \text { weeks } \# \#\end{array}$ & $\mathrm{~N}=229$ & $\mathrm{~N}=230$ \\
\hline Hyperuricaemia & $22(9.6)$ & $29(12.6)$ \\
\hline Increased AST & $8(3.5)$ & $20(8.7)$ \\
\hline Increased $\gamma$-glutamyltransferase & $3(1.3)$ & $12(5.2)$ \\
\hline Increased leukocytes & $4(1.7)$ & $10(4.3)$ \\
\hline Increased ALT & $5(2.1)$ & $9(3.9)$ \\
\hline Hyperglycaemia & $6(2.6)$ & $7(3.0)$ \\
\hline QTcF ms & $\mathrm{N}=232$ & $\mathrm{~N}=232$ \\
\hline$>450-480$ & $27(11.6)$ & 36 (15.5) \\
\hline$>480-500$ & $3(1.3)$ & $5(2.2)$ \\
\hline$>500$ & $1(0.4)$ & $2(0.9)$ \\
\hline
\end{tabular}

Data are presented as $\mathrm{n}(\%)$. $\mathrm{n}$ : number of patients with AE category; $\mathrm{N}$ : number of patients with data; TB: tuberculosis; AST: aspartate aminotransferase; ALT: alanine aminotransferase; QTcF: Fridericia-corrected QT. " : in the six patients, this was due to vomiting, TB, pregnancy, QT prolonged (QTcF $461 \mathrm{~ms}$, change from reference of $11 \mathrm{~ms}$ ), inadequate control of diabetes and hallucinations; ${ }^{1}$ : highlighted due to their potential importance based on nonclinical and clinical data on bedaquiline and identified using the Standardised MedDRA Queries (SMQs) for acute pancreatitis, rhabdomyolysis/myopathy, severe cutaneous AEs and Torsades de Pointes/QT prolongation, and selected sub-SMQs from drug-related hepatic disorders (liver-related investigations, signs and symptoms (SMQ), liver-related coagulation and bleeding disturbances (SMQ) and cholestasis and jaundice of hepatic origin (SMQ), including hepatic failure, fibrosis and cirrhosis and other liver damage-related conditions (SMQ) and hepatitis, noninfectious (SMQ)); ${ }^{+}$: no incidences of Torsades de Pointes or ventricular arrhythmias were reported; $\S_{\text {: }}$ in all nine patients who experienced the severe cutaneous SMQ event, this was based upon a preferred term of conjunctivitis lall events were of grade 1 and 2, and two $(0.9 \%)$ were reported as possibly related to bedaquiline by the investigator); $f:$ in patients who experienced the acute pancreatitis SMQ event, this was based upon investigations (blood amylase increased, blood bilirubin increased), hepatobiliary disorders (hyperbilirubinaemia), and metabolism and nutrition disorders (hyperamylasaemia); \#\#: includes all laboratory abnormalities not only those reported as an AE.

Among the 57 (27.8\%) nonresponders at week 120, 24 (11.7\%) discontinued after culture conversion, 25 (12.2\%) had failed to convert and eight (3.9\%) had reverted to culture-positive (figure 4), with a median time to reversion of 382 days after baseline. At study completion, 125/205 mITT patients (61.0\%) were "cured", 3/205 (1.5\%) completed, 32/205 (15.6\%) failed treatment, 31/205 (15.1\%) transferred out/ defaulted and 14/205 (6.8\%) died on the basis of the modified WHO outcome definitions for MDR-TB [21] (figure 4). 


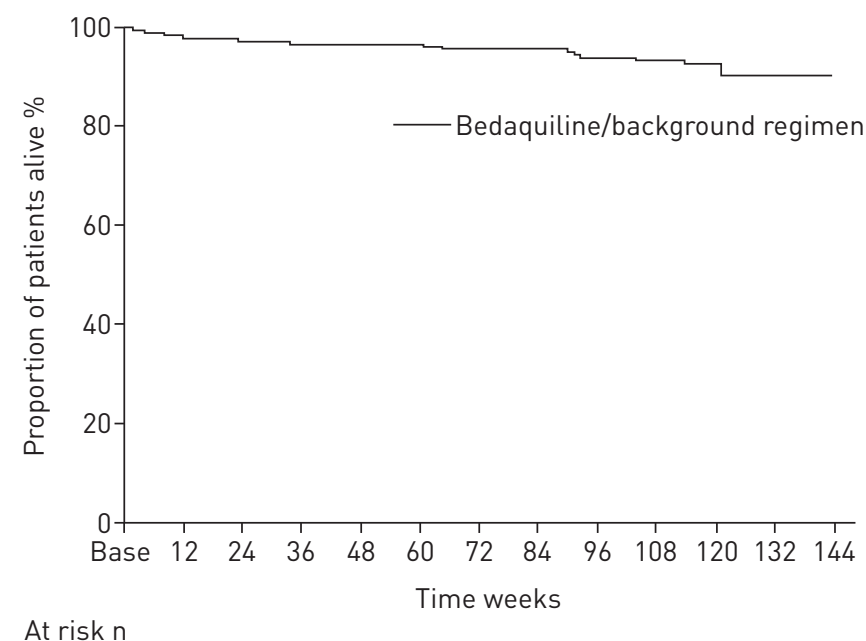

$233227221217216216213211202194 \quad 117 \quad 9 \quad 0$

FIGURE 1 Kaplan-Meier plot for time to death (safety (intent-to-treat) population). Base: baseline.

Conversion rates were $73.1 \%$ (95\% exact CI 62.9-81.8\%) in patients with MDR-TB (resistance limited to isoniazid and rifampin), $70.5 \%$ (95\% exact CI 54.8-83.3\%) in patients with pre-XDR-TB and 62.2\% (95\% exact CI 44.8-77.6\%) in patients with XDR-TB. All 23 patients with XDR-TB who culture-converted and completed the study were responders at 120 weeks. Although some of these patients were still receiving BR at the week 120 visit, the majority (16/23) were not and had a median of 5.4 months of treatment-free follow-up. Therefore, in a least some patients with XDR-TB, 6 months of bedaquiline given with an optimised BR followed by continuation of a BR alone contributed to sputum culture conversion and prevention of early relapse 6 months after stopping treatment. There was also no clear relationship between baseline bedaquiline MIC and culture conversion at end-point.

None of the 93 patients with MDR-TB at baseline developed pre-XDR-TB during the trial, but of the 44 patients with pre-XDR-TB at baseline, three nonresponders (all three with resistance to ofloxacin) developed XDR-TB. Baseline DST showed the bedaquiline MIC of M. tuberculosis isolates ranged from

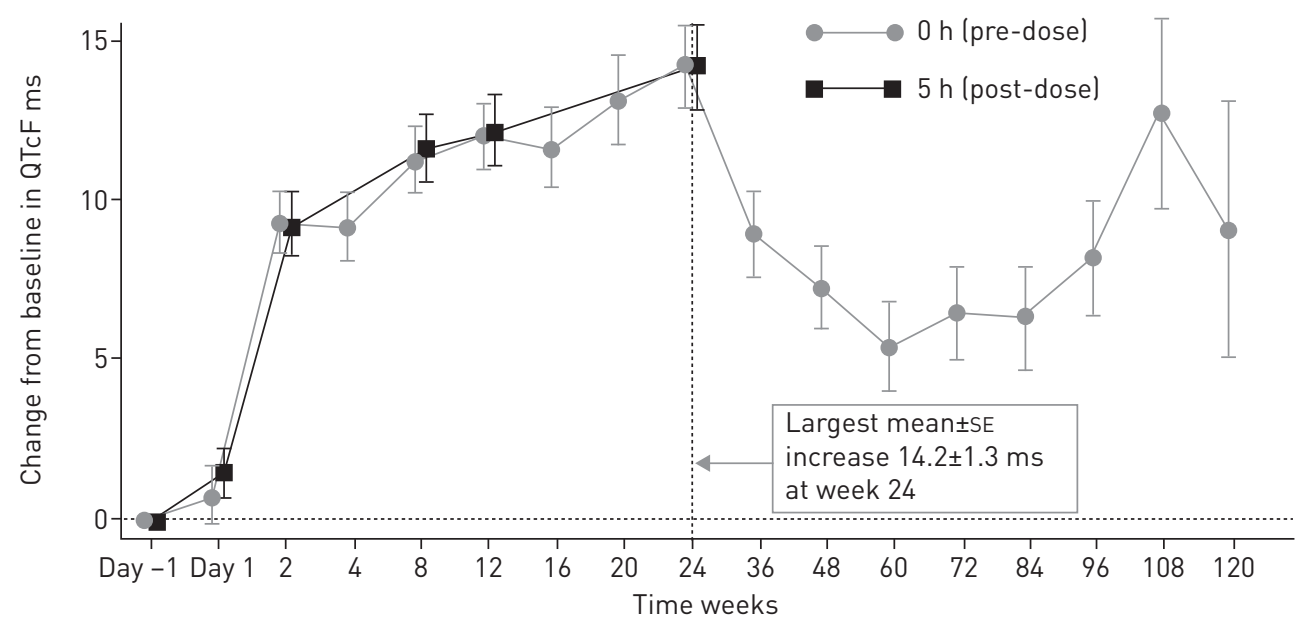

Patients $\mathrm{n}$
$\mathrm{Oh}$ 228 $227 \quad 220$ 221218216

FIGURE 2 Fridericia-corrected QT (QTcF) changes from reference (safety (intent-to-treat) population). Data are presented as mean \pm SE. The wide error bars after week 96 are explained by the small number of patients still on treatment at these times and only patients on treatment had ECG assessments $(n=48$ at week 108 and $\mathrm{n}=30$ at week 120). The incremental increase between weeks 60 and 108 was not considered clinically relevant due to the wide standard error and the limited number of patients assessed. 
FIGURE 3 Kaplan-Meier plot for time to confirmed culture conversion (efficacy (modified intent-to-treat) population). The vertical line represents the median time to culture conversion. Base: baseline.

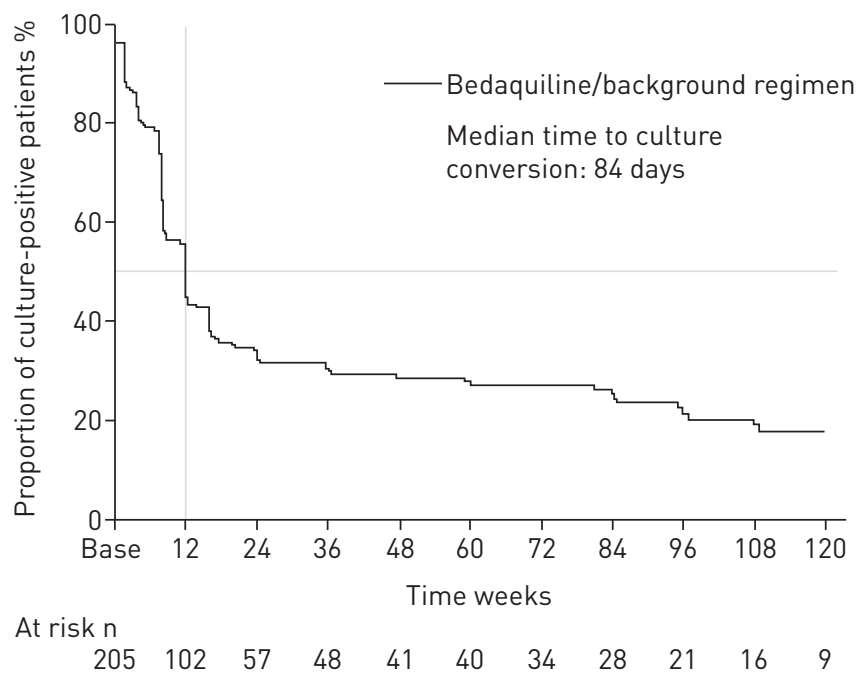

Study definition (missing=failure)

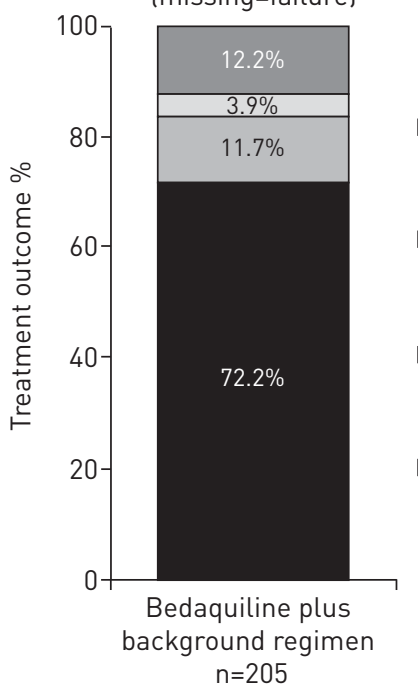

Failed to convert

$\square$ Reverted to positive

$\square$ Discontinued after conversion

- Conversion

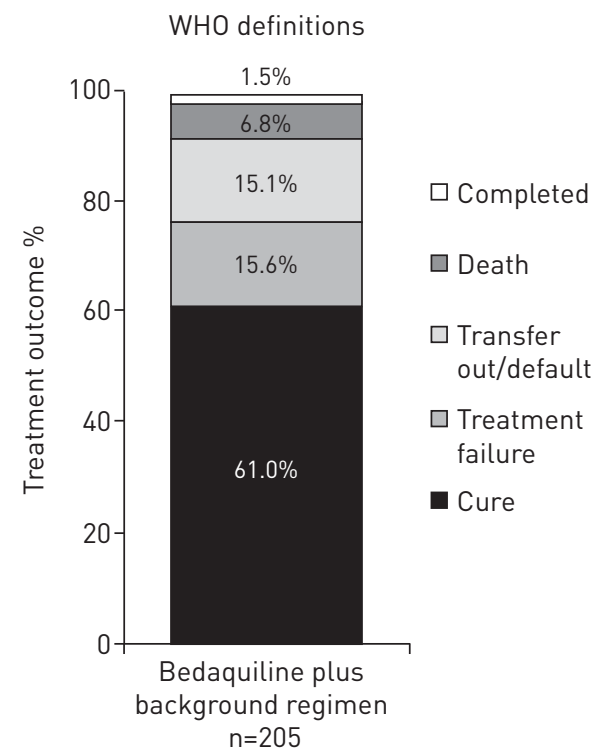

FIGURE 4 Week 120 outcomes in the efficacy (modified intent-to-treat) population based on the protocol-defined analysis method (missing=failure) and the modified World Health Organization (WHO) outcome definitions for multidrug-resistant tuberculosis [21]. Cure: A patient who completed the study and was consistently culture-negative (with at least five consecutive negative cultures from samples collected at least 30 days apart) for at least the final 12 months of the study. If only one positive culture was reported during that time, a patient was still considered cured, provided that this positive culture was followed by a minimum of three consecutive negative cultures taken at least 30 days apart. Treatment failure: a patient who completed the study and was not cured as per definition above. Transfer out/default: a patient who discontinued the study for any reason. Death: a patient who died during the study. Patients who died during survival follow-up before or within the week 120 window were also included in this category. Patients who died during survival follow-up after the week 120 window were not included in this category as the event occurred after the planned duration (120 weeks) of the trial. Completed: a patient who completed the study but did not meet the definition for cure or treatment failure due to lack of bacteriologic results.

0.0075 to $0.24 \mu \mathrm{g} \cdot \mathrm{mL}^{-1}$ for $97.0 \%$ of strains. 24 patients in the efficacy population had repeat DST for bedaquiline at week 24 or later. 12 of these had a post-baseline $\geqslant 4$-fold increase in bedaquiline MIC and the paired isolates were confirmed to be the same strain using 24-locus mycobacterial interspersed repetitive unit variable number tandem repeat (MITU-VNTR) genotyping; nine of the 12 had MIC $\geqslant 0.24 \mu \mathrm{g} \cdot \mathrm{mL}^{-1}$ and eight had MIC $>0.48 \mu \mathrm{g} \cdot \mathrm{mL}^{-1}$. Post-baseline increases in bedaquiline MIC were almost exclusively seen in patients with pre-XDR-TB (4/12) and XDR-TB (7/12). None of these post-baseline isolates had a polymorphism in the ATP operon, but all 12 had mutations in Rv0678 post-baseline [25]. 
TABLE 4 Summary of deaths (safety (intent-to-treat) population)

\section{While on 120 -week study}

$\mathrm{N}=233$

\section{During survival follow-up of premature discontinuations \\ $\mathrm{N}=\mathbf{2 0}$}

Deaths $\mathbf{n}^{\#}$

Microbiology response

Investigator-reported causes of death

\author{
Completed 24 weeks of bedaquiline \\ treatment $\mathbf{n}$ with full exposure/n \\ total deaths \\ Median time since last bedaquiline \\ intake days \\ Investigator causality (related to \\ bedaquiline) $n / n$ total deaths
}

\section{2}

7 nonconverters; 4 converters; 1 reverted to positive?

TB-related illness ( $n=5)$; haemoptysis $(n=1)$; renal impairment $(n=1)$; lung infection $(n=1)$; pneumonia $(n=1)$; respiratory failure $(n=1)$; congestive cardiac failure $(n=1)$; hypertension $(n=1)$

$$
9 / 12
$$

12/12 not related ${ }^{+}$, including 1 doubtfully related (renal impairment)
3 nonconverters; 0 converters 1 reverted to positive ?

TB-related illness ( $\mathrm{n}=4)$

$1 / 4$

4/4 not related ${ }^{+}$including 1 doubtfully related (TB-related illness)

TB: tuberculosis; QTcF: Fridericia-corrected QT. " : none of the patients who died had QTcF prolongation $>500 \mathrm{~ms}$ or had grade 3 or 4 liver adverse events (AEs) identified by the Standardised MedDRA Queries (SMQs) for hepatic AEs or AEs identified by the SMQ for acute pancreatitis; П: patient whose sputum became culture-positive after initial sputum conversion; ${ }^{+}$: not related or doubtfully related deaths were summarised as not related to bedaquiline.

Five of 12 patients with $\geqslant 4$-fold increase in bedaquiline MIC at 24 weeks or later had treatment success at end-point, against three of five patients who were still culture-positive at 24 weeks with $<4$-fold increase in bedaquiline MIC.

\section{Discussion}

The primary objective of this study was to acquire further safety data on bedaquiline in a larger, more heterogeneous population. A key finding of this study was that overall mortality was $6.9 \%$, as compared with the previously reported mortality rate of $12 \%$ in stage 2 of C208 [11]. This $6.9 \%$ mortality rate compares favourably with other cohorts (e.g. 15\% in a large meta-analysis [4]) and included rigorous survival verification of patients who had withdrawn from the study. Unsurprisingly, mortality was largely accounted for by respiratory system pathology. All but one patient who died from TB or a TB-related illness had either not converted or reverted to positive. No evidence was found for a causal relationship between bedaquiline and mortality.

The most common AEs were similar to those frequently described in treatment cohorts with MDR-TB $[22,23]$. In the current study, individual AEs were generally reported less frequently and were of decreased severity compared with C208 [11]. This could be due to inclusion of patients in the current study with prior MDR-TB treatment who agreed to be retreated. No new safety signals related to bedaquiline were noted in this larger trial. Increases in hepatic transaminases have previously been reported with bedaquiline [11], but the $6.4 \%$ incidence of hepatic-related AEs was less than that reported in other MDR-TB cohorts [26]. Only two patients had clinically significant QTcF prolongation ( $>500 \mathrm{~ms}$ ), and both were taking clofazimine and one also had hypokalaemia. More intensive ECG and electrolyte monitoring is therefore advisable when bedaquiline is co-administered with clofazimine.

The proportion of patients with confirmed culture conversion in C209 was similar to C208 at weeks 24 (79.5\% versus $78.8 \%$ in $\mathrm{C} 208)$ and 120 (72.2\% versus $62.1 \%)$ [11]. The median time to sputum culture conversion was shorter in C209 probably because the majority of patients were receiving second-line therapy at baseline. The cure rate based on the modified WHO outcome definitions for MDR-TB [21] was $61.0 \%$ versus $57.6 \%$ in $\mathrm{C} 208$ and the durable efficacy of bedaquiline treatment to 120 weeks was also consistent with C208 [11], strengthening the observation that the 24-week culture conversion rate is predictive for long-term MDR-TB treatment outcome. Any comparison between the study outcomes needs to consider differences in patient populations. The C209 study included a lower proportion of patients infected with HIV (4.0\% versus $10.1 \%)$ and with cavitation $(63.5 \%$ versus $75.9 \%)$ than in C208, but critically also included patients with XDR-TB (16.3\%), a category of MDR-TB with particularly poor outcomes especially when there is HIV co-infection [5-7, 27]. 
In this study, culture conversion was similar for patient subgroups with MDR-TB (resistance limited to isoniazid and rifampin) and pre-XDR-TB (73.1\% versus $70.5 \%)$, but was numerically lower in the XDR-TB subgroup (62.2\%). With caution in comparing across studies, these observed response rates are higher than others have reported $[5-8,27,28]$, but consistent with response rates reported in compassionate-use programmes with bedaquiline $[13,15]$. No patients with XDR-TB who converted during the trial relapsed. Overall, these results indicate that bedaquiline, added to a patient's BR, resulted in good culture conversion rates in a broad range of patients, including the majority of patients with XDR-TB.

Despite extensive prior treatment and the inclusion of patients with pre-XDR-TB (21.5\%), progression to XDR-TB (by acquisition of additional resistance) was infrequent and only detected in three patients with pre-XDR-TB at baseline. This is compatible with the C208 study [11] where fewer patients receiving bedaquiline acquired additional drug resistance compared with those receiving placebo and supports that inclusion of bedaquiline in MDR-TB regimens prevents development of resistance to important companion drugs. Increases in bedaquiline MICs occurred infrequently, and were almost exclusively seen in patients with pre-XDR and XDR-TB. Importantly, all 12 patients who had a post-baseline $\geqslant 4$-fold increase in bedaquiline MIC had mutations in Rv0678, a transcriptional repressor of the MmpS5-MmpL5 efflux pump [25]. Recently, this novel efflux mechanism of resistance has been identified to result in low-level resistance to bedaquiline and clofazimine $[25,29,30]$. The current data do not suggest that increases in bedaquiline MICs due to mutations in Rv0678 have a negative effect on treatment response.

The single-arm design and the lack of HIV-positive patients receiving ART are limitations of this study. Another limitation is that in 55/233 patients with resistance to isoniazid and rifampicin, resistance to fluoroquinolones or second-line injectable drugs (pre-XDR or XDR status) was not determined. Further studies are required to confirm the safety of bedaquiline in patients receiving ART. The open-label study design and inclusion criteria were chosen to enrol a large study population more representative of clinical practice. Nevertheless, the low mortality versus other MDR-TB cohorts [4], absence of unexpected adverse drug reactions and high clinical response rate support the conclusion that bedaquiline can be used safely to strengthen MDR-TB and XDR-TB treatment regimens. The rapid culture conversion rate and high overall treatment response also suggest that combining bedaquiline with an optimised BR can reduce the period when person-to-person transmission of MDR-TB may occur. A phase 3 study (STREAM stage 2; www.clinicaltrials.gov: NCT02409290) will enrol a larger number of patients and evaluate 9 months of bedaquiline given with a "modified Bangladesh regimen" in a controlled design [31, 32]. Combining bedaquiline with other drugs (approved or currently in clinical development) also has the potential to deliver a more efficacious regimen for patients with more resistant subtypes of MDR, such as XDR-TB or subtypes of XDR [8]. These new drugs and regimens are expected to be an important component in the new WHO global strategy focussed on TB elimination [33-35]. However, additional safety studies (e.g. evaluation of QT prolongation when bedaquiline and delamanid are combined will be investigated in ACTG A5343) may be required alongside appropriately powered efficacy studies (e.g. STREAM stage 2).

\section{Acknowledgements}

Medical writing support was provided by Ian Woolveridge of Zoetic Science, an Ashfield company, Macclesfield, UK; this support was funded by Janssen Pharmaceuticals.

We would like to thank the patients and their families, investigators, study centre staff, and Janssen study personnel, in particular Chrispin Kambili and Els De Paepe, for their input into this manuscript. We also acknowledge the contributions of David McNeeley who previously worked for Janssen Pharmaceuticals.

The TMC207-C209 Study Group included the following Principal Investigators: Francesca Conradie, Xiaohong Chen, Naihui Chu, Charoen Chuchottaworn, Manfred Danilovits, Jorge De Los Rios, Andreas Diacon, Dina Diaz, Vladislav Erokhin (Irina Vasilyeva), Victor Flores, Sung Chul Hwang, Carlos Iberico, Boris Kazennyi, Young Sam Kim, Anu Kurve, Mykhaylo Kuzhko, O Jung Kwon, Vaira Leimane, Svetlana Lyepshina, Andrey Maryandyshev, Vasiliy Melnyk, Ronelle Narasimooloo (Alexander Pym), Lihua Qiu, Gönenç Ortaköylü, Seref Ozkara, Rafael Reano, Tae Sun Shim, Shen-Jie Tang, Eduardo Ticona Chavez, Tawatchai Wiwatworapan, Xia Zhang.

\section{References}

1 World Health Organization. Global Tuberculosis Report 2014. http://apps.who.int/iris/bitstream/10665/137094/1/ 9789241564809_eng.pdf?ua=1 Date last accessed: May 5, 2015.

2 Shah NS, Wright A, Bai G-H, et al. Worldwide emergence of extensively drug-resistant tuberculosis. Emerg Infect Dis 2007; 13: 380-387.

3 Banerjee R, Allen J, Westenhouse J, et al. Extensively drug-resistant tuberculosis in California, 1993-2006. Clin Infect Dis 2008; 47: 450-457.

4 Ahuja SD, Ashkin D, Avendano XM, et al. Multidrug resistant pulmonary tuberculosis treatment regimens and patient outcomes: an individual patient data meta-analysis of 9,153 patients. PLoS Med 2012; 9: e1001300.

5 Dheda K, Shean K, Zumla A, et al. Early treatment outcomes and HIV status of patients with extensively drug-resistant tuberculosis in South Africa: a retrospective cohort study. Lancet 2010; 375: 1798-1807.

6 O'Donnell MR, Padayatchi N, Kvasnovsky C, et al. Treatment outcomes for extensively drug-resistant tuberculosis and HIV co-infection. Emerg Infect Dis 2013; 19: 416-424. 
Falzon D, Gandhi N, Migliori GB, et al. Resistance to fluoroquinolones and second-line injectable drugs: impact on multidrug-resistant TB outcomes. Eur Respir J 2013; 42: 156-168.

8 Migliori GB, Sotgiu G, Gandhi NR, et al. Drug resistance beyond extensively drug-resistant tuberculosis: individual patient data meta-analysis. Eur Respir J 2013; 42: 169-179.

9 Andries K, Verhasselt P, Guillemont J, et al. A diarylquinoline drug active on the ATP synthase of Mycobacterium tuberculosis. Science 2005; 307: 223-227.

10 World Health Organization. The use of bedaquiline in the treatment of multidrug-resistant tuberculosis. Interim policy guidance (WHO/HTM/TB/2013.6). http://apps.who.int/iris/bitstream/10665/84879/1/9789241505482_eng. pdf Date last accessed: May 5, 2015.

11 Diacon AH, Pym A, Grobusch MP, et al. Multidrug-resistant tuberculosis and culture conversion with bedaquiline. N Engl J Med 2014; 371: 723-732.

12 Tiberi S, De Lorenzo S, Centis R, et al. Bedaquiline in MDR/XDR-TB cases: first experience on compassionate use. Eur Respir J 2014; 43: 289-292.

13 Guglielmetti L, Le Dû D, Jachym M, et al. Compassionate use of bedaquiline for the treatment of multidrug-resistant and extensively drug-resistant tuberculosis: interim analysis of a French cohort. Clin Infect Dis 2015; 60: 188-194.

14 Udwadia ZF, Amale RA, Mullerpattan JB. Initial experience of bedaquiline use in a series of drug-resistant tuberculosis patients from India. Int J Tuberc Lung Dis 2014; 18: 1315-1318.

15 Ndjeka N, Conradie F, Schnippel K, et al. Treatment of drug-resistant tuberculosis with bedaquiline in a high HIV prevalence setting: an interim cohort analysis. Int J Tuberc Lung Dis 2015; 19: 979-985.

16 Diacon AH, Pym A, Grobusch MP, et al. The diarylquinoline bedaquiline for multidrug-resistant tuberculosis. $N$ Engl J Med 2009; 360: 2397-2405.

17 Diacon AH, Donald PR, Pym A, et al. Randomized pilot trial of eight weeks of bedaquiline (TMC207) treatment for multidrug-resistant tuberculosis: long-term outcome, tolerability, and effect on emergence of drug resistance. Antimicrob Agents Chemother 2012; 56: 3271-3276.

18 World Health Organization. Guidelines for the programmatic management of drug-resistant tuberculosis. Emergency update 2008 (WHO/HTM/TB/2008.402). http://whqlibdoc.who.int/publications/2008/9789241547581_ eng.pdf Date last accessed: May 5, 2015.

19 National Institute of Allergy and Infectious Diseases, Division of Microbiology and Infectious Diseases (DMID) adult toxicity table, November 2007. www.niaid.nih.gov/LabsAndResources/resources/DMIDClinRsrch/Documents/ dmidadulttox.pdf Date last accessed: May 5, 2015.

20 Medical Dictionary for Regulatory Activities (MedDRA). Standardised MedDRA queries. http://www.meddra.org/ standardised-meddra-queries Date last accessed: May 5, 2015.

21 World Health Organization. Treatment of tuberculosis: guidelines. 4th Edn (WHO/HTM/TB/2009.420). www. aidsdatahub.org/dmdocuments/Treatment_of_TB_Guidelines.pdf Date last accessed: May 5, 2015.

22 Bloss E, Kuksa L, Holtz TH, et al. Adverse events related to multidrug-resistant tuberculosis treatment, Latvia, 2000-2004. Int J Tuberc Lung Dis 2010; 14: 275-281.

23 Sagwa E, Mantel-Teeuwisse AK, Ruswa N, et al. The burden of adverse events during treatment of drug-resistant tuberculosis in Namibia. South Med Rev 2012; 5: 6-13.

24 Shen X, Yuan Z, Mei J, et al. Anti-tuberculosis drug-induced liver injury in Shanghai: validation of Hy's Law. Drug Saf 2014; 37: 43-51.

25 Andries K, Villellas C, Coeck N, et al. Acquired resistance of Mycobacterium tuberculosis to bedaquiline. PLoS One 2014; 9: e102135.

26 Keshavjee S, Gelmanova IY, Shin SS, et al. Hepatotoxicity during treatment for multidrug-resistant tuberculosis: occurrence, management and outcome. Int J Tuberc Lung Dis 2012; 16: 596-603.

27 Pietersen E, Ignatius E, Streicher EM, et al. Long-term outcomes of patients with extensively drug-resistant tuberculosis in South Africa: a cohort study. Lancet 2014; 383: 1230-1239.

28 Falzon D, Mirzayev F, Wares F, et al. Multidrug-resistant tuberculosis around the world: what progress has been made? Eur Respir J 2015; 45: 150-160.

29 Hartkoorn RC, Uplekar S, Cole ST. Cross-resistance between clofazimine and bedaquiline through upregulation of MmpL5 in Mycobacterium tuberculosis. Antimicrob Agents Chemother 2014; 58: 2979-2981.

30 Gupta S, Cohen KA, Winglee K, et al. Efflux inhibition with verapamil potentiates bedaquiline in Mycobacterium tuberculosis. Antimicrob Agents Chemother 2014; 58: 574-576.

31 Van Deun A, Maug AK, Salim MA, et al. Short, highly effective, and inexpensive standardized treatment of multidrug-resistant tuberculosis. Am J Respir Crit Care Med 2010; 182: 684-692.

32 Aung KJ, Van Deun A, Declercq E, et al. Successful '9-month Bangladesh regimen' for multidrug-resistant tuberculosis among over 500 consecutive patients. Int J Tuberc Lung Dis 2014; 18: 1180-1187.

33 D'Ambrosio L, Dara M, Tadolini M, et al. Tuberculosis elimination: theory and practice in Europe. Eur Respir J 2014; 43: 1410-1420.

34 Diel R, Loddenkemper R, Zellweger JP, et al. Old ideas to innovate tuberculosis control: preventive treatment to achieve elimination. Eur Respir J 2013; 42: 785-801.

35 Lönnroth K, Migliori GB, Abubakar I, et al. Towards tuberculosis elimination: an action framework for low-incidence countries. Eur Respir J 2015; 45: 928-952. 